\title{
Likelihood of attending to the color word modulates Stroop interference
}

\author{
Yang Seok Cho • Jong Moon Choi • Robert W. Proctor
}

Published online: 7 December 2011

(C) Psychonomic Society, Inc. 2011

\begin{abstract}
Three experiments investigated whether the Stroop color-naming effect is modulated by the likelihood of a color word capturing visual attention. In Experiment 1, a bar or a neutral word was presented at fixation as a color carrier, along with a color word randomly appearing in either an achromatic color (white in the main experiment, gray in a follow-up) or purple. Reduction of the Stroop effect (known as Stroop dilution) occurred when the color word was achromatic but not (or to a lesser extent) when it was in purple. In Experiment 2, the color of the color word remained constant throughout trial blocks, and Stroop dilution was equally evident when the word was always in purple and when it was always in white. In Experiment 3, a color bar was presented as the color carrier with both a color word and a neutral word. In this case, the Stroop effect was larger when the color word appeared in purple, and smaller when the neutral word appeared in purple, than when neither word did. These results imply that the extent to which processing of a color word occurs is determined by the likelihood of the word capturing attention.
\end{abstract}

Keywords Stroop - Selective attention Attentional capture

\footnotetext{
Y. S. Cho $(\bowtie)$

Department of Psychology, Korea University,

Anam-Dong Seongbuk-Gu,

Seoul 136-701, Korea

e-mail: yscho_psych@korea.ac.kr

J. M. Choi

Department of Psychology, University of Maryland,

College Park, MD, USA

R. W. Proctor

Department of Psychological Sciences, Purdue University,

West Lafayette, IN, USA

Task performance is often affected by irrelevant stimulus information, which indicates a failure of selective attention. The Stroop effect has been taken to be a good example of such failure (MacLeod, 1991; Stroop, 1935/1992). In the Stroop color-naming task, participants are to say the color of a target stimulus in the presence of an irrelevant color word (which may carry the target color or be separate from it). Responses are slower and less accurate when the to-beignored color word conflicts with the task-relevant color information than when it does not. The Stroop effect is often cited as evidence that word recognition is automatic (e.g., Brown, Roos-Gilbert, \& Carr, 1995), with the logic being that the color word affects task performance because it is automatically recognized (e.g., MacLeod \& Dunbar, 1988). However, the Stroop effect has been investigated mainly through indirect measures of word recognition (i.e., the influences of color words on the time to name the taskrelevant colors), without consideration of how visual attention is deployed. Consequently, the conclusion that visual attention is not required for recognition of the color word may be questioned (Choi, Cho, \& Proctor, 2009).

Control of visual attention involves both bottom-up and top-down mechanisms (Johnson \& Proctor, 2004). Visual attention is guided to a salient stimulus by the bottom-up mechanism (e.g., Theeuwes, 1992) or to a stimulus defined as relevant to the assigned task by the top-down mechanism (e.g., Francolini \& Egeth, 1979). Folk, Remington, and Wright (1994) showed, by manipulating the type of spatial cue, that top-down control settings affect whether attention will be captured by a salient stimulus. A cuing effect was obtained when the spatial cue included a feature related to the target-defining feature (e.g., a rotating cue for a rotating target), but not when the spatial cue was unrelated to the target-defining feature (e.g., an abrupt-onset cue for a rotating target). Folk et al. (1994) pointed out that even salience- 
based selectivity includes the top-down control mechanism and does not operate in a purely bottom-up manner.

Becker, Folk, and Remington (2010) recently elaborated the view that attentional capture is contingent on the goals of the performer, providing evidence that stimuli that are similar to the target can capture attention better than can the designated target if those stimuli are more distinct from the other distractors. For example, when searching for an orange target among yellow distractors, a red stimulus will capture attention more than will the orange target, because it differs more from the yellow distractors. Flowers and Dutch (1976) reported similar results for versions of the Stroop task that required participants to search lists for target colors: Search times were longer and the Stroop effect larger when the possible targets were dissimilar (e.g., orange, green, purple) rather than similar (e.g., red, orange, yellow) in hue. Many accounts of attentional capture, including that of contingent capture, assume that visual attention is guided to locations in order of decreasing activation, as determined by both bottomup and top-down mechanisms (see Wolfe, 1994). In the Stroop task, top-down processing involves being prepared to attend and respond to the color of a stimulus from the set of possible target colors (e.g., red, yellow, green, or blue). The possibility exists that the color word in the Stroop task may be recognized without intention because visual attention is involuntarily directed to it as a consequence of these top-down processes interacting with the bottomup processes (Lachter, Forster, \& Ruthruff, 2004).

\section{Orientation of visual attention in the Stroop task}

In the single-stimulus version of the Stroop task (see, e.g., Dalrymple-Alford \& Budayr, 1966), in which a colored color word is displayed, visual attention is oriented to the color word because it contains the task-relevant information (color) and is the only stimulus in the display. In a twostimulus version of the Stroop task, a typical procedure is to present a color bar at fixation and a color word in white (on a dark background) or black (on a white background) immediately above or below the color bar (e.g., Kahneman \& Chajczyk, 1983). Because the color bar is the salient and task-relevant stimulus (Cho, Lien, \& Proctor, 2006; Herd, Banich, \& O'Reilly, 2006), visual attention would be oriented to the color bar by both bottom-up and top-down control mechanisms. Since, in the two-stimulus version, the color word is typically presented in an achromatic white or black color that is distinct from the set of possible target colors, it has a lower probability of capturing attention. Hence, the Stroop effect is typically smaller in the two- than in the one-stimulus version (MacLeod, 1991).

In one study using the two-stimulus version, Kahneman and Chajczyk (1983, Exp. 1) presented an additional color- neutral word on some trials, along with the color bar and the color word (e.g., if the color word was above the bar, the neutral word was below the bar). The Stroop effect obtained when the color word and the neutral word were both presented was about half the size of that obtained when only the color word accompanied the color bar. Kahneman and Chajczyk called this effect Stroop dilution and attributed it to attentional capture by the neutral word, based on the assumption that word recognition in the Stroop task requires attention. According to them, only one word at a time can capture visual attention, and when two words are presented, each word has an equal chance of capturing attention. If the color word captures visual attention on a trial, that word is identified, and Stroop interference occurs. However, if the neutral word attracts attention, the color word is not identified, resulting in no Stroop effect.

In line with Kahneman and Chajczyk (1983), Cho, Lien, and Proctor (2006) suggested that color-word processing is modulated by allocation of visual attention (see also MacLeod \& Bors, 2002). In Cho et al.'s Experiment 1, the Stroop dilution effect was not obtained when the color word was presented as a color carrier and a neutral word (in white) appeared above or below it. However, when the color word (in white) was displayed as a distractor, with a neutral word or a bar presented as the color carrier, the Stroop dilution effect was obtained, regardless of whether the location of the color carrier was constant or varied across the three stimulus positions.

On the basis of these results, Cho et al. (2006) suggested that the Stroop dilution effect is due to a shift of attention from the color carrier to a distractor. According to Cho et al., the color carrier is most likely to capture attention first because of the top-down contingent capture by color (e.g., Ansorge, Horstmann, \& Carbone, 2005). After initial processing of the color carrier, attention is shifted to a distractor, if possible. Thus, when the color word was the color carrier, there was no Stroop dilution effect because the color word always captured attention. When the color word was distinct from the color carrier, visual attention had less chance to shift to the color word if the color carrier was a neutral word than if it was a bar because more time was required to process the target color on a neutral word than on a bar. In other words, because the color word was presented in an achromatic white color, distinct from the potential target colors, attention was first captured by the color carrier stimulus and then had less chance to shift to the color word when the color carrier was a neutral word rather than a bar.

Roberts and Besner (2005) reported similar results. In their Experiment 2, in which a color word (in white) was presented above or below a color carrier presented at fixation, a 4-ms Stroop effect was obtained when a neutral word was the color carrier, as compared to a 54-ms Stroop effect when a color bar was the carrier. In their Experiment 5, in which the 
color word was presented at fixation as the color carrier, a Stroop effect of more than $100 \mathrm{~ms}$ was obtained, regardless of whether a neutral word was presented as a distractor. Roberts and Besner also found that the Stroop effect was not reduced when the color carrier was a string of symbols, Xs, or digits as compared to when it was a color bar. However, when the color carrier was a string of consonants or a repeated word, the Stroop effect disappeared (i.e., the Stroop effect was diluted).

Unlike Cho et al. (2006), Roberts and Besner (2005) suggested that Stroop dilution occurs because attentional resources for processing multiple stimuli in the same domain (e.g., alphabetic characters) are limited. They referred to this explanation as the domain-specific, or material-specific, limited-capacity account. According to it, when the color word is presented as a distractor, processing of that word depends on the nature of the material at fixation. Roberts and Besner placed emphasis on the symbolic content of that material, noting, for example, that "neutral words and consonant strings interfered with processing of a color word distractor more than characters from the top of the keyboard" (p. 5). If the material at fixation is from the same domain as the color word (i.e., alphabetic characters), the attentional resources for processing the color word are unavailable because of high processing load (Lavie, 2005; Lavie \& Fox, 2000), resulting in no Stroop effect. If the material at fixation is from a different domain (e.g., nonalphabetic characters), the attentional resources for processing the color word are available because of low processing load, resulting in a large Stroop effect.

\section{Present study}

Cho et al.'s (2006) attentional capture account and Roberts and Besner's (2005) material-specific limited-capacity account of the Stroop dilution effect agree that attention is required for color-word recognition, but they offer different perspectives on the influence of the nature of the color carrier. Cho et al.'s account suggests that the Stroop effect is reduced when the color carrier is a neutral word because the probability that the color word will be attended is less than when the color carrier is a bar. That is, according to this account, for both color carrier types, attention is attracted initially to the color carrier on most trials because the achromatic color in which the color word is displayed is distinct from the set of possible target colors. However, since more time is needed to process the target color when the carrier is a neutral word rather than a bar, attention is less likely to shift subsequently to the color word, resulting in a reduced Stroop effect. An implication is that if the color word is displayed in a color that is less distinct from the set of possible target colors, attention will be directed initially toward the color word on more trials, reducing the Stroop dilution effect. In contrast, Roberts and Besner suggested that the size of the Stroop effect depends on whether the color carrier is composed from the same alphabetic material as the color word. Because material-specific attentional resources are limited, the color word is less well recognized when the color carrier is a neutral word, which is in the same alphabetic domain, than when it is a color bar, which is in a different domain. By this account, distinctiveness from the target set of the color in which the color word is displayed should be of little consequence.

Thus, a way to test between the attentional capture and material-specific limited-capacity accounts is to vary the similarity of the color in which the color word is presented to the colors of the target color set. As noted, prior studies have displayed the color word in an achromatic white or black to make it distinct from the stimulus carrying the target color. The likelihood of attentional capture by the color word can be increased, though, by presenting it in a chromatic color that is similar to, but not included in, the set of target colors. The color purple is more similar perceptually than is white to the set of possible target colors (red, yellow, green, and blue) used in the present study. Therefore, the attentional capture account predicts that competition for visual attention between the color carrier and the color word will be more likely to occur for trials on which the color word is in purple rather than white (e.g., Duncan \& Humphreys, 1989). If the likelihood of the color word attracting visual attention is a determinant of Stroop effect size, then Stroop dilution should be less evident when the color word is in purple rather than white because of this difference in attentional competition. This prediction of reduced Stroop dilution when the distractor appears in purple is in contrast to that of the material-specific limited-capacity account, according to which the amount of Stroop dilution should not depend on whether the color word appears in purple or white.

We conducted three experiments using this distractor color variable to evaluate whether the size of the Stroop effect depends on the probability of the color word capturing visual attention. In Experiment 1, we used a procedure in which a target color was presented on a color bar or on a neutral word at fixation, with a color word presented above or below the color carrier. The crucial difference from previous studies was that the color word was presented in white on only a random half of the trials, and was presented in purple on the other half.

Experiment 2 was similar to Experiment 1, but for any given participant, the color word appeared in a constant color, either purple or white, throughout the experiment. This allowed us to determine whether the influence of the presentation color of the color word on the Stroop effect was due to attentional competition or color-color interference (Glaser \& Glaser, 1982; i.e., color naming being hindered by a distractor stimulus of incongruent color that is presented 
along with the target color). When the color of the color word does not vary from trial to trial, the color should lose its ability to capture visual attention (see, e.g., Melara \& Mounts, 1993). If so, there should be no competition for attention between the color carrier and the color word. Thus, the size of the Stroop effect should be determined only by the nature of the color carrier, resulting in a Stroop dilution effect regardless of whether the color word was displayed in purple or white. In contrast, if color-color interference contributes to the Stroop effect, the Stroop effect should still be modulated by the color of the color word in a manner similar to Experiment 1.

In Experiment 3, we sought to obtain converging evidence for the attentional capture hypothesis by investigating whether the Stroop effect is modulated by the color of the distractor when the discriminability of the color carrier from the color word is high. To avoid the contribution of the perceptual discriminability of the color carrier from the color word, the color carrier was always a bar at fixation, with a color word presented above the color carrier and a neutral word presented below it, or vice versa. One of the words was in purple and the other in white, or both words were in white. Because visual attention is more likely to be attracted to the purple stimulus than to the white stimulus, if visual attention is required for processing the meaning of the color word, the Stroop effect should be largest when the color word is presented in purple. Also, the Stroop effect should be smallest when the neutral word is presented in purple.

\section{Experiment 1}

Experiment 1 was conducted to obtain evidence about whether Stroop dilution depends on the likelihood that the color word will capture visual attention, as Cho et al. (2006) suggested, or on limited-capacity material-specific processing resources for multiple stimuli in the same (alphabetic) domain, as Roberts and Besner (2005) suggested. To manipulate the probability of attentional capture by the color word, this word was presented in one of two colors, white or purple, which differed from the target colors (blue, green, yellow, and red). According to Folk, Remington, and Johnston (1992), visual attention is more likely to be oriented involuntarily to a stimulus that shares a critical physical property with the target than to one that does not. Thus, the color word presented in purple would have more chance of attracting visual attention than would the color word presented in white, because of the former's greater physical similarity to the members of the set of target colors.

In the present experiment, a color word was located above or below the color carrier, which was a bar or a neutral word presented at fixation. The color word appeared in white on half of the trials and in purple on the remainder.
When the color word was presented in purple, competition for attention between the color carrier and the color word would be expected to occur because of top-down contingent capture by color (i.e., the purple distractor would be more likely to capture visual attention). If the size of the Stroop effect depends on the probability of the color word capturing visual attention, a Stroop dilution effect should occur when the color word appears in white (because the probability of attention being directed to it is higher with a bar as the color carrier than with a neutral word). However, there should be less dilution of the Stroop effect when the color word appears in purple (because the material of the color carrier would have little or no influence on the attentional competition between the color carrier and the color word). In contrast to this prediction, if the magnitude of the Stroop effect is modulated by the limited capacity of attentional resources in the alphabetic domain, dilution of the Stroop effect should occur regardless of whether the color word is presented in purple or white, because the word's color does not alter its alphabetic nature.

\section{Method}

Participants A group of 10 undergraduate students at Korea University participated to fulfill their course requirement. All had normal or corrected-to-normal visual acuity and color vision.

Stimuli and apparatus Stimuli were presented on the CRT monitor (17 in.) of a personal computer. The distance between the monitor and the participant was approximately 60 $\mathrm{cm}$. The stimuli were presented by E-Prime software (Version 1.2, Psychology Software Tools, Pittsburgh, PA). Vocal responses were recorded by a microphone through a response box operated with E-Prime software. The microphone was positioned between the participant and the monitor.

A target color was presented as the color of a bar $\left(0.76^{\circ} \times\right.$ $\left.1.87^{\circ}\right)$ or the font color of a neutral word $\left(0.76^{\circ} \times 1.60^{\circ}\right)$, along with a color word $\left(0.76^{\circ} \times 1.60^{\circ}\right)$ on a black background. The target colors were red $(\mathrm{R}=255, \mathrm{G}=0, \mathrm{~B}=0)$, yellow $(R=255, G=255, B=0)$, green $(R=0, G=168, B=$ $20)$, and blue $(R=0, G=0, B=255)$, with their appearances corresponding to the following CIE color coordinates: red, $x=$ 41.24, $y=21.26, z=1.93$; yellow, $x=77, y=92.78, z=$ 13.85; green, $x=13.129, y=28.056, z=5.332$; blue, $x=$ $18.05, y=7.22, z=95.05$. The colors were not equated for luminance, but had values of $8.34,34.64,10.84$, and $4.13 \mathrm{~cd}$ for red, yellow, green, and blue, respectively.

The color word was 빨강 [palgang] ("red" in Korean), 노랑 [nolang] ("yellow" in Korean), 초록 [cholok] ("green" in Korean), or 파랑 [palang] ("blue" in Korean) and was presented in either white $(\mathrm{R}=255, \mathrm{G}=255, \mathrm{~B}=255$; CIE color coordinates: $x=95.05, y=100, z=108.90 ; 33.98 \mathrm{~cd}$ ) or 
purple $(\mathrm{R}=170, \mathrm{G}=0, \mathrm{~B}=170$; $\mathrm{CIE}$ color coordinates: $x=$ $26.35, y=12.66, z=43.11 ; 4.86 \mathrm{~cd})$. The neutral word was 중심 [joongsim] ("center" in Korean), 전기 [jeonki] ("electricity" in Korean), 함성 [hamsung] ("shout" in Korean), or 향수 [hyangsoo] ("perfume" in Korean), which correspond to the word frequencies of the color words. The distance between target and distractor was $0.57^{\circ}$.

Procedure Participants performed the experiment individually under dim light. A total of 16 practice trials were performed before 256 test trials, with half of the trials being congruent and half incongruent. The stimulus presentation on a trial is depicted in Fig. 1. Each trial started with a fixation point for $500 \mathrm{~ms}$, followed by a blank display for $800 \mathrm{~ms}$. After the blank display, a forward-masking display, a target display, and a backward-masking display were successively presented for $250 \mathrm{~ms}$ each. The masking displays consisted of three rows of four white Xs, which covered the places where the color carrier and color word would appear. Participants were told to name the color of the color carrier verbally and to ignore the other stimuli. The color carrier was a bar on half of the trials and a neutral word on the rest; the carrier occurred at the center position on all trials, with the color word located in the upper position on half of the trials and the lower position on the other half. After a response, an intertrial interval (ITI) of $1,300 \mathrm{~ms}$ preceded onset of the fixation point for the next trial. If the response was incorrect or no response was made, the ITI was preceded by a $300-\mathrm{ms}$ feedback tone. The color word was presented in white or purple with equal probability. All experimental conditions were intermixed and randomly presented.

\section{Results}

RTs $<150 \mathrm{~ms}$ or $>1,500 \mathrm{~ms}(0.47 \%$ of the total trials $)$ were removed as outliers. All analyses were performed without these outliers. The mean RT and percent error (PE) were $553 \mathrm{~ms}$ and $1.53 \%$, respectively. Mean RT and PE data for each participant were calculated as a function of distractor color (white or purple), carrier type (neutral word or bar), and congruency (incongruent or congruent). Analyses of variance (ANOVAs) with all variables analyzed within subjects were conducted on the mean RT and PE data (see Table 1 for condition means). The $\alpha$ level for all statistical analyses in this study was .05 .
Table 1 Experiment 1 (and follow-up): Mean reaction times, in milliseconds, and percentages of errors (in parentheses) as a function of color of the color word, color carrier, and congruency

\begin{tabular}{llll}
\hline $\begin{array}{l}\text { Color of Color Word } \\
\text { and Color Carrier }\end{array}$ & Congruency & \multirow{2}{*}{ Stroop Effect } \\
\cline { 2 - 3 } & Congruent & Incongruent & \\
\hline $\begin{array}{l}\text { Experiment } 1 \\
\text { White }\end{array}$ & & & \\
Color bar & $506(0.94)$ & $561(1.90)$ & $55(0.96)$ \\
Neutral word & $556(2.51)$ & $574(0.94)$ & $18(-1.57)$ \\
Purple & & & \\
Color bar & $521(1.29)$ & $558(3.45)$ & $37(2.16)$ \\
Neutral word & $556(0.94)$ & $592(0.31)$ & $36(-0.63)$ \\
Follow-Up Experiment & & & \\
Gray & & & $78(2.81)$ \\
Color bar & $540(0.00)$ & $618(2.81)$ & $25(0.63)$ \\
$\quad$ Neutral word & $601(1.25)$ & $626(1.88)$ & \\
Purple & & & $52(4.12)$ \\
Color bar & $560(0.31)$ & $612(4.43)$ & $28(0.94)$ \\
Neutral word & $608(0.94)$ & $636(1.88)$ & \\
\hline
\end{tabular}

Stroop effect $=$ Incongruent minus Congruent

$R T$ The main effect of carrier type was significant, $F(1,9)=$ 119.67, $M S E=180, p<.0001$. RTs were shorter when the color carrier was a bar $(M=537 \mathrm{~ms})$ than when it was a neutral word $(M=569 \mathrm{~ms})$. There was also a main effect of congruency, $F(1,9)=44.56, M S E=606, p<.0001$. Responses were faster when the color word was congruent with the color of the color carrier $(M=535 \mathrm{~ms})$ than when it was incongruent $(M=571 \mathrm{~ms})$, showing a 36-ms Stroop effect. The Stroop effect was modulated by carrier type, $F(1,9)=5.79, M S E=298, p=.0395$, which was $46 \mathrm{~ms}$ when the color carrier was a bar, $F(1,9)=71.11, M S E=$ $299, p<.0001$, and $27 \mathrm{~ms}$ when it was a neutral word, $F(1,9)=25.32, M S E=299, p=.0007$, showing a Stroop dilution effect.

The main effect of distractor color was not significant, $F(1,9)=2.01, M S E=594, p=.1895$, although RTs tended to be shorter when the color word was presented in white $(M=549 \mathrm{~ms})$ rather than purple $(M=557 \mathrm{~ms})$. Also, distractor color did not interact with carrier or congruency, $F_{\mathrm{s}}(1,9)<1.0$. Most importantly, the interaction of distractor
Fig. 1 Example of a sequence of events on a trial in Experiments 1 and 2. The upper word is printed in purple and the lower word in yellow in the stimulus display

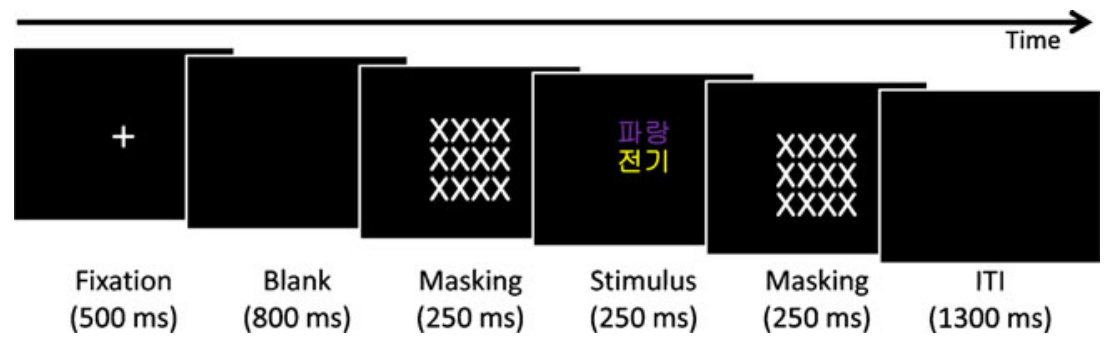

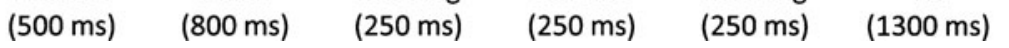


color, carrier type, and congruency was significant, $F(1,9)=$ $8.72, M S E=184, p=.0162$. To decompose this interaction, the data were analyzed for the white and purple color words separately. With the color word presented in white, the Stroop effect was larger when the color carrier was a bar $(55 \mathrm{~ms})$ than when it was a neutral word $(18 \mathrm{~ms}), F(1,9)=$ $11.30, M S E=295, p=.0084$. Further analysis showed that the Stroop effect for each color carrier was significant, $F \mathrm{~s}(1,9)=51.57$ and $5.90, p \mathrm{~s}=.0001$ and .0381 . With the color word presented in purple, however, the Stroop effect was of similar size, regardless of whether the color carrier was the bar $(37 \mathrm{~ms})$ or the neutral word $(36 \mathrm{~ms})$. Thus, unlike when the color word was in white, as in previous studies, no Stroop dilution effect was evident when the word was in purple.

A further analysis showed that the difference in the result patterns for the white and purple distractor words also was evident in the RT distributions. We performed RT bin analyses in which the individual-participant RT distributions for each cell of the design (distractor color, carrier type, and congruency) were partitioned into quarters and then analyzed with bin as an additional factor (see Proctor, Miles, \& Baroni, 2011, for a review of the method). Of interest are the terms that involve the variables of bin and congruency, which indicate changes in the Stroop effect across the RT distribution. The two-way interaction of congruency and bin was significant, $F(3,27)=32.14, M S E=$ 213, $p<.0001$, showing that the Stroop effect increased across the RT distribution - that is, effects of 18, 30, 40, and $61 \mathrm{~ms}$ at Bins 1-4. More importantly, this interaction was qualified by a four-way interaction involving the additional variables of distractor color and carrier type, $F(3,27)=2.94$, $M S E=163, p=.051$. When the color word was in white, there was a $\mathrm{Bin} \times$ Congruency interaction, $F(3,27)=35.18$, $M S E=109, p<.0001$, and a three-way interaction of those variables with carrier type, $F(3,27)=3.47, M S E=336, p=$ .0297: The Stroop effect increased as RT increased when the color carrier was a bar $(25,44,58$, and $95 \mathrm{~ms}), F(3,27)=$ $16.9, M S E=264, p<.0001$, and much less so when the color carrier was a neutral word $(3,20,22$, and $27 \mathrm{~ms})$, $F(3,27)=2.92, M S E=181, p=.0519$. When the color word was in purple, there was a Bin $\times$ Congruency interaction, $F(3,27)=12.47, M S E=252, p<.0001$, but no threeway interaction of those variables with carrier type, $F<1$ : The Stroop effect increased as RT increased to similar extents when the color carrier was a bar $(18,30,45$, and $65 \mathrm{~ms}), F(3,27)=5.69, M S E=359, p=.0038$, and when it was a neutral word $(25,26,33$, and $59 \mathrm{~ms}), F(3,27)=4.82$, $M S E=262, p=.0082$.

$P E$ An ANOVA similar to the primary analysis for RTs showed that the color of the color word interacted with carrier type, $F(1,9)=5.93, M S E=21.04, p=.0376$, and carrier type also interacted with congruency, $F(1,9)=$ $18.28, M S E=35.28, p=.0021$. The Stroop effect was $1.56 \%$ when the color carrier was a bar, and $-1.10 \%$ when the carrier was a neutral word. Simple main effect analyses showed that the positive Stroop effect when the carrier was a bar and the negative Stroop effect when the carrier was a neutral word were both significant, $F \mathrm{~s}(1,9)=12.57$ and 6.25, MSEs $=24.26$ and 12.07, $p \mathrm{~s}=.0063$ and .0338 , respectively. There was no other significant effect.

\section{Discussion}

If Stroop dilution occurs because of the limited processing capacity for alphabetic material, the Stroop effect should have been smaller when the color carrier was a neutral word than when it was a bar, regardless of whether the color word was presented in white or purple. When the color word was presented in white, as in the studies of Cho et al. (2006) and Roberts and Besner (2005), the Stroop effect was smaller and the increase across the RT distribution less if the color carrier was a neutral word than if it was a bar, indicating the diluting influence of the neutral word. However, when the color word was presented in purple, no dilution of the Stroop effect by the neutral word was evident in the overall Stroop effect or in its change across the RT distribution, suggesting that in this case the color word competed with the color carrier to attract visual attention to a similar extent, regardless of the presence of a neutral word. This novel result of the absence of Stroop dilution in RTs when the color word was presented in purple occurred even though the neutral-word color carrier was in the same alphabetic domain as the color word and was different from the nonalphabetic domain of the bar color carrier. Moreover, the Stroop effect tended to be larger with the color word presented in purple $(36 \mathrm{~ms})$ than in white (18 ms) when the color carrier was a neutral word, $F(1,9)=4.02, M S E=193.69, p=.0760$, suggesting that the extent to which a color word is recognized depends on the chance that the color word will be attended rather than on the nature of the color carrier itself.

It should be noted that, when the color carrier was a bar, the Stroop effect was smaller when the color word was presented in purple $(37 \mathrm{~ms})$ rather than white $(55 \mathrm{~ms})$, $F(1,9)=7.71, M S E=107.16, p=.0215$. This result is most likely due to the opportunity for the color word to capture attention differing as a function of the word's color. When the color word was presented in white, the size of the Stroop effect was determined by the probability of an attention shift from the color carrier to the color word after processing of the color carrier, which was relatively high when the color carrier was a bar. On the contrary, when the color word was presented in purple, either the word or the bar could initially capture attention. When the bar captured attention, a subsequent attention shift to the purple color word might occur less often because of 
its being less distinctive than a white color word. This possibility is consistent with the Stroop effect being smaller in the last RT bin for purple color words than for white ones.

A couple of additional factors that differed between the white and purple color words could have influenced the sizes of the Stroop effects that they produced: The preand postmasks were white and might have masked the white color words more effectively, and the luminance of the white color words was higher than that of the purple color words. However, any account of the main results that is based on these factors would be called into question by the fact that the relative influences of the purple and white color words on performance were opposite for the bar and neutral-word color carriers: The Stroop effect was larger for color words appearing in white rather than purple when the color carrier was a bar, but smaller for color words appearing in white rather than purple when the color carrier was a neutral word. This interaction ruled out any simple account in terms of the relative visibility of the color words in white as opposed to purple.

To assess more directly whether the result pattern in Experiment 1 was due mainly to the luminance differences between white and purple, another 10 participants were tested under identical conditions but with the white color words and masks replaced by gray ones $(\mathrm{R}=85, \mathrm{G}=$ $85, \mathrm{~B}=85$; CIE color coordinates: $x=10.560, y=$ $11.111, z=12.10 ; 4.86 \mathrm{~cd}$ ) equal in luminance to the purple color words. That experiment showed a Stroop dilution effect of $39 \mathrm{~ms}, F(1,9)=20.17, M S E=383, p=.0015$, that interacted with distractor color, $F(1,9)=5.30, M S E=$ $201, p=.0469$, since the Stroop effect was larger for the gray distractor stimuli $(52 \mathrm{~ms})$ than for the purple ones $(24$ $\mathrm{ms}$ ). Although the purple distractors showed some Stroop dilution in this follow-up experiment, the $28-\mathrm{ms}$ difference in effect size from that shown by the gray distractors was similar to the 36-ms difference in Experiment 1. The similarity of results is emphasized by the fact that ANOVAs on $\mathrm{RT}$ and PE that included experiment as a factor showed no significant terms involving experiment, $p \mathrm{~s}>.09$.

Although our hypothesis is that the critical factor differentiating the results for the purple and white color words is the greater similarity of the color purple to the set of possible target colors, purple might exert its effect more locally, mainly on trials for which the target color is most similar to purple (i.e., when the target color is red or blue). To evaluate this possibility, we performed ANOVAs for Experiment 1 and the follow-up study with the factors described above but with an additional factor of the four target colors (see Table 2 for means). For both experiments, the three-way interaction of distractor color, carrier type, and congruency (indicative of Stroop dilution being less with the purple than with the white distractor color) was significant, $F \mathrm{~s}(1,9)=7.55$ and $8.04, M S E \mathrm{~s}=901$ and 611, $p \mathrm{~s}<.025$.
Table 2 Experiment 1 (and follow-up, in parentheses): Mean reaction times, in milliseconds, as a function of target color, color of the color word, color carrier, and congruency

\begin{tabular}{|c|c|c|c|}
\hline \multirow{2}{*}{$\begin{array}{l}\text { Color of Color Word } \\
\text { and Color Carrier }\end{array}$} & \multicolumn{2}{|l|}{ Congruency } & \multirow[t]{2}{*}{ Stroop Effect } \\
\hline & Congruent & Incongruent & \\
\hline \multicolumn{4}{|l|}{ Target Color: Red } \\
\hline \multicolumn{4}{|l|}{ White (Gray) } \\
\hline Color bar & $483(530)$ & $562(603)$ & $79(73)$ \\
\hline Neutral word & $545(586)$ & $555(586)$ & $10(0)$ \\
\hline \multicolumn{4}{|l|}{ Purple } \\
\hline Color bar & $512(552)$ & $539(573)$ & $27(21)$ \\
\hline Neutral word & $532(604)$ & $586(605)$ & $54(1)$ \\
\hline \multicolumn{4}{|l|}{ Target Color: Yellow } \\
\hline \multicolumn{4}{|l|}{ White (Gray) } \\
\hline Color bar & $493(537)$ & $533(605)$ & $40(68)$ \\
\hline Neutral word & $548(596)$ & $559(610)$ & $11(11)$ \\
\hline \multicolumn{4}{|l|}{ Purple } \\
\hline Color bar & $516(539)$ & $548(608)$ & $32(69)$ \\
\hline Neutral word & $548(600)$ & $571(613)$ & $23(13)$ \\
\hline \multicolumn{4}{|l|}{ Target Color: Green } \\
\hline \multicolumn{4}{|l|}{ White (Gray) } \\
\hline Color bar & $507(538)$ & $581(636)$ & $74(98)$ \\
\hline Neutral word & $558(604)$ & $575(638)$ & $17(34)$ \\
\hline \multicolumn{4}{|l|}{ Purple } \\
\hline Color bar & $512(567)$ & $572(608)$ & $60(41)$ \\
\hline Neutral word & $569(597)$ & $599(648)$ & $30(51)$ \\
\hline \multicolumn{4}{|l|}{ Target Color: Blue } \\
\hline \multicolumn{4}{|l|}{ White (Gray) } \\
\hline Color bar & $538(553)$ & $578(637)$ & $40(84)$ \\
\hline Neutral word & $571(615)$ & $608(665)$ & $37(37)$ \\
\hline \multicolumn{4}{|l|}{ Purple } \\
\hline Color bar & $546(582)$ & $580(665)$ & $34(83)$ \\
\hline Neutral word & $577(632)$ & $613(682)$ & $36(50)$ \\
\hline
\end{tabular}

However, the four-way interaction with target color did not attain the .05 level, $F_{\mathrm{s}}(3,27)=2.36$ and $1.69, M S E \mathrm{~s}=848$ and $1,086, p \mathrm{~s}=.094$ and .194 , indicating that there was no significant difference in the dilution effect across the four target colors. Separate three-way ANOVAs for each distractor color were performed to follow up this trend, and in neither case was the three-way interaction of target color, carrier type, and congruency significant, $F_{\mathrm{s}}(3,27)=2.11$ and $1.94, p \mathrm{~s}>.12$, for white and purple distractors, respectively. Separate three-way ANOVAs for the follow-up experiment also showed no interaction of target color, carrier type, and congruency effect for each distractor color, $F(3,27)=1.32$ and $F(3,27)<1.0, p s>.28$, for white and purple distractors, respectively. The nonsignificant trends in the data across the two experiments were for the Stroop dilution effect to be largest for red targets and least for 
blue targets, whereas on the basis of similarity to the distractor color purple, both should have shown larger dilution effects than the dissimilar yellow and green target colors.

Another question one might ask is whether the difference in processing for distractors presented in purple versus white in Experiment 1 was evident only when the distractor color was the same as on the previous trial, therefore not requiring a switch in task set (see Kiesel et al., 2010, for a review). However, an ANOVA with prior distractor color as an additional factor showed that this variable did not enter into any interaction that approached the .05 level, indicating little influence on performance of the distractor color from the previous trial. One might also argue that the lack of Stroop dilution effect with the color word presented in purple was due to the nontarget color interfering with the colorword processing. That is, it is possible that the colorword processing was degraded because of a need to inhibit the processing of the nontarget color (Folk \& Remington, 1998). If this were the case, though, the Stroop effect should also have been larger with the color word presented in white than in purple when the color carrier was a neutral word.

\section{Experiment 2}

Experiment 2 was conducted to determine whether the nontarget color degraded color-word recognition by some means other than attracting visual attention. Although it has been reported that an irrelevant color did not produce interference with responding to a relevant color stimulus when the additional color did not correspond to a response (Glaser \& Glaser, 1982; La Heij, Helaha, \& Van den Hof, 1993), there was still a possibility that the additional purple color could have interfered with color-word recognition in Experiment 1. To test this possibility, the color of the color word was manipulated as a between-subjects variable: The color word was displayed in white for half of the participants and in purple for the other half. This invariance of the task-irrelevant color for individual participants throughout the experiment should cause it to lose its attentional competition ability (see, e.g., Morein-Zamir, Henik, \& SpitzerDavidson, 2002). Thus, attentional capture by the color word should be easily avoided by the cognitive-control mechanism. If the nontarget color does not interfere with color-word recognition, the size of the Stroop effect should be identical, regardless of whether the color word is presented in white or purple. Moreover, the Stroop effect should be diluted when the color carrier is a neutral word. On the contrary, if the nontarget color affects word recognition, Stroop interference should be more evident when the color word is presented in white than in purple, as it was in Experiment 1
Method

Participants A group of 20 new undergraduate students at Korea University participated to fulfill a course requirement. All reported having normal or corrected-to-normal visual acuity and color vision.

Stimuli, apparatus, and procedure The stimuli, apparatus, and procedure were identical to those in Experiment 1, with the exception that the color of the color word was white for 10 participants and purple for the other 10 participants.

\section{Results}

In total, $0.59 \%$ of the trials were removed, using the same RT cutoff criteria as in Experiment 1. All statistical analysis was done without these outliers. The mean RT and PE were $564 \mathrm{~ms}$ and $2.21 \%$, respectively. Mean RT and PE data for each participant were calculated as a function of distractor color (white or purple), carrier type (neutral word or bar), and congruency (congruent and incongruent); see Table 3 for the group means. ANOVAs were conducted on the mean RT and PE data, with Distractor Color as a between-subjects factor and the other factors analyzed within subjects.

$R T$ The main effect of carrier type was significant, $F(1,18)=$ $152.63, M S E=134, p<.0001$. Mean RTs were shorter when the color carrier was a bar $(M=548 \mathrm{~ms})$ than when it was a neutral word $(M=580 \mathrm{~ms})$. The main effect of congruency was also significant, $F(1,18)=80.33, M S E=295, p<$ .0001 . Responses were faster when the color word was congruent with the color of the color carrier $(M=547 \mathrm{~ms})$ than when it was incongruent $(M=581 \mathrm{~ms})$, showing a 34$\mathrm{ms}$ Stroop effect. The interaction of congruency with carrier type was significant, $F(1,18)=33.57, M S E=204, p<$ .0001 , with the Stroop effect being larger when the color carrier was a bar (53 ms), $F(1,18)=137.33, M S E=204, p<$

Table 3 Experiment 2: Mean reaction times, in milliseconds, and percentages of errors (in parentheses) as a function of color of the color word, color carrier, and congruency

\begin{tabular}{llll}
\hline $\begin{array}{l}\text { Color of Color Word } \\
\text { and Color Carrier }\end{array}$ & Congruency & Stroop Effect \\
\cline { 2 - 3 } & Congruent & Incongruent & \\
\hline White & & & \\
Color bar & $529(1.41)$ & $583(6.02)$ & $54(4.61)$ \\
Neutral word & $576(1.57)$ & $591(1.90)$ & $15(0.32)$ \\
Purple & & & \\
Color bar & $515(0.32)$ & $567(3.32)$ & $52(3.00)$ \\
Neutral word & $568(1.43)$ & $585(1.75)$ & $17(0.32)$ \\
\hline
\end{tabular}

Stroop effect $=$ Incongruent minus Congruent 
.0001 , rather than a neutral word $(16 \mathrm{~ms}), F(1,18)=12.43$, $M S E=204, p=.0024$. Most importantly, neither distractor color nor its interaction with any other term was significant, $F \mathrm{~s}(1,18) \leq 2.70, p \mathrm{~s} \geq .1175$. The sizes of the Stroop and Stroop dilution effects were similar for the white and purple distractors.

The similarity of the effects for white and purple colorword distractors in Experiment 2 extended to the RT distributions. As in Experiment 1, we performed an RT distribution analysis based on dividing the distributions into four bins. Also as in Experiment 1, the two-way interaction of bin and congruency was significant, $F(3,54)=20.89, M S E=$ $231, p<.0001$, but unlike in the previous experiment, the four-way interaction with the variables of distractor color and carrier type was not significant, $F<1$. When the color carrier was a bar, the Stroop effect increased as RT increased, regardless of whether the color word was presented in white $(21,38,53$, and $102 \mathrm{~ms}), F(3,27)=11.64, M S E=$ $602, p<.0001$, or purple $(24,39,57$, and $90 \mathrm{~ms}), F(3,27)=$ $14.28, M S E=258, p<.0001$. When the color carrier was a neutral word, the Stroop effect varied little across bins, again regardless of whether the color word was presented in white $(7,18,21$, and $14 \mathrm{~ms}), F(3,27)=1.45, M S E=137$, $p=.2508$, or purple $(20,20,18$, and $12 \mathrm{~ms}), F(3,27)<1.0$.

$P E$ The main effect of carrier type was significant, $F(1,18)=$ $5.28, M S E=4.62, p=.0337$, as was that of congruency, $5 F$ $(1,18)=26.86, M S E=3.17, p<.0001$. Errors were fewer when the meaning of the color word was congruent with the color of the color carrier $(M=1.18 \%)$ than when it was incongruent $(M=3.25 \%)$, showing a $2.07 \%$ Stroop effect. The interaction between congruency and carrier type was significant, $F(1,18)=15.77, M S E=3.84, p=.0009$. The PE Stroop effect was a significant $3.8 \%$ when the color carrier was a bar, $F(1,18)=144.63, M S E=3.84, p<.0001$, but a nonsignificant $0.32 \%$ when the color carrier was a neutral word, $F(1,18)<1.0$.

The effect of distractor color was not significant, $F(1,18)=$ 2.21, MSE $=9.39, p=.1540$. Although the interaction of distractor color and carrier type approached significance, $F(1,18)=3.32, M S E=4.62, p=.0853$, there were no other interactions of the distractor color with other terms, $F \mathrm{~s}(1,18) \leq 1.01, p \mathrm{~s} \geq .3284$.

\section{Discussion}

Unlike in Experiment 1, in which the color of the distractor varied randomly from trial to trial, here the Stroop dilution effect was equally evident, regardless of whether the color word was presented in white or purple, when the color of the color word was held constant for a given participant in Experiment 2 . For both white and purple color words, when the color carrier was a bar, the Stroop effect increased monotonically across the RT distribution; but when the carrier was a neutral word, the Stroop effect remained small across the RT distribution. Because of the invariance of the color of the distractor, the color word lost its ability to compete with the color carrier for visual attention even when the color word was presented in purple. Thus, the probability of the color word capturing attention was modulated only by whether the color carrier was a bar or a neutral word, resulting in Stroop dilution for both purple and white color words. These results imply that the modulation of the Stroop effect by the color of the color word in Experiment 1 was due to attentional competition between the color carrier and the color word, and not to color-color interference.

Note that the lack of significant effects of distractor color in this experiment provides additional evidence against the possibility that the difference in Stroop dilution for white and purple color words in Experiment 1 was due to a difference in the words' visibility. Even though in Experiment 2 the mask characters were white and the white color words were of higher luminance than the purple color words, the two colors yielded similar results when each participant received only one of the colors.

Our finding that the distractor apparently did not attract attention when it was purple on all trials is different from a result obtained by Ansorge and Heumann (2004, Exp. 1c) in a peripheral cuing task for which the constant color of an uninformative cue (yellowish red) was similar to that of the target stimulus (red). In their experiment, the task was to respond to target location (left or right of fixation); the cue and target were identical disks that were the same except for their colors, and the cue onset 68 or $85 \mathrm{~ms}$ prior to the target in either the same or opposite position. Responses were $43 \mathrm{~ms}$ faster when the cue and target positions coincided than when they did not, suggesting that attention was captured by the cue. Although their study differed in many respects from ours, the possibly critical variables include the facts that cue onset always preceded target onset, that all target events were of a single color, and that target color was irrelevant to the task of responding to target location. Moreover, the cuing effect in that experiment was half the size of the effect in their Experiment $1 \mathrm{~b}(83 \mathrm{~ms})$, which differed mainly in mixing trials with the similar cue with trials on which the cue was in a color (bluish green) dissimilar to that of the target. Thus, in their study, attentional capture by the similar cue seems to have been reduced when its color was constant versus when it was not.

\section{Experiment 3}

In Experiment 1, the Stroop effect was larger with a color word that appeared in white rather than purple when the 
color carrier was a bar, even though the opposite pattern of results was obtained when the color carrier was a neutral word. These results are somewhat inconsistent with the prediction made by the attentional capture account. However, as mentioned earlier, the probability of the color word capturing attention was modulated by different factors in Experiment 1, in which either a neutral word or a bar was presented as the color carrier, resulting in low discriminability of the color carrier from the color word presented in purple. When the color word was presented in white, the magnitude of the Stroop effect was mainly modulated by whether the color carrier was a bar or a neutral word. However, when the color word was presented in purple, attentional competition could occur between the color carrier and the color word because of the low discriminability of the color carrier from the color word.

Experiment 3 was conducted to investigate whether the Stroop effect is modulated by the color of the distractor when the discriminability of the color carrier is high. To increase the discriminability of the color carrier in this experiment, it was always a bar presented at fixation, and a color word and a neutral word were presented separately as distractors, one above the color bar and the other below it. Either the color word, the neutral word, or neither was presented in purple, and otherwise they were presented in white. Thus, the color carrier could be easily discriminated from the words even when one of them was presented in purple, because the color carrier and the distractors were from different (nonalphabetic and alphabetic) stimulus domains and appeared reliably at different positions. Consequently, attention could be expected to orient initially to the color carrier. After the color-carrier processing, an attentional shift would be more likely to occur to the distractor presented in purple rather than white. If the disappearance of Stroop dilution was due to the nontarget color attracting visual attention, the Stroop effect should increase when the color word was presented in purple and decrease when the neutral word was presented in purple, relative to when both were presented in white.

\section{Method}

Participants A group of 10 new undergraduate students at Korea University participated to fulfill a course requirement. All reported having normal or corrected-to-normal visual acuity and color vision.

Apparatus, stimuli, and procedure The apparatus, stimuli, and procedure were identical to those of Experiment 1, with the following exceptions. A bar was always presented at fixation as the color carrier. A color word and a neutral word were located above and below the color carrier. On a third of the trials, the color word was presented in purple and the neutral word in white. On another third of the trials, the neutral word was presented in purple and the color word in white. On the remaining trials, the color word and neutral word were both presented in white. Twelve practice trials preceded 180 test trials.

\section{Results}

In total, $0.94 \%$ of the trials were removed, using the same RT cutoff criteria as in the previous experiments. The overall mean RT and PE were $583 \mathrm{~ms}$ and $1.30 \%$, respectively. Mean RT and PE data were calculated for each participant as a function of word presented in color (colored word: none, color word, or neutral word) and congruency (congruent or incongruent); see Table 4 for the group means. ANOVAs were conducted on the mean RT and PE data, with those variables analyzed as within-subject factors.

$R T$ The main effect of colored word did not reach significance, $F(1,9)=1.89, M S E=357, p=.1799$, but the main effect of congruency did, $F(1,9)=45.67, M S E=897, p<$ .0001 . Responses were faster when the color word was congruent with the color of the color bar $(M=557 \mathrm{~ms})$ than when it was not $(M=609 \mathrm{~ms})$, showing a $52-\mathrm{ms}$ Stroop effect. Also, the Stroop effect was modulated as a function of the colored word, $F(2,18)=8.88, M S E=168, p=.0021$, being largest when the color word was in purple $(68 \mathrm{~ms})$, intermediate when neither word was in purple $(56 \mathrm{~ms})$, and smallest when the neutral word was in purple $(33 \mathrm{~ms})$. A Scheffé test with Stroop effect size as the dependent variable revealed that the Stroop effect was smaller when the neutral word was presented in purple than when either the color word was presented in purple or both words were in white. There was no significant difference between the latter two conditions.

An RT bin analysis similar to those performed for Experiments 1 and 2 showed a Bin $\times$ Congruency interaction, $F(3,27)=15.07, M S E=1,005, p<.0001$, with the Stroop effect increasing from Bins 1 to 4 , with values of 22, 40, 54, and $97 \mathrm{~ms}$, respectively. The three-way interaction of those variables with colored word was also significant, $F(6,54)=$

Table 4 Experiment 3: Mean reaction times, in milliseconds, and percentages of errors (in parentheses) as a function of target location, color of the color word, color carrier, and congruency

\begin{tabular}{llll}
\hline \multirow{2}{*}{ Word Shown in Purple } & \multicolumn{2}{c}{ Congruency } & \multirow{2}{*}{ Stroop Effect } \\
\cline { 2 - 3 } & Congruent & Incongruent & \\
\hline None & $550(0.67)$ & $606(1.34)$ & $56(0.67)$ \\
Color word & $548(0.00)$ & $616(2.73)$ & $68(2.73)$ \\
Neutral word & $573(0.00)$ & $606(3.04)$ & $33(3.04)$ \\
\hline
\end{tabular}

Stroop effect $=$ Incongruent minus Congruent 
2.91, MSE $=465, p<.0156$. The increase was largest when color word was in purple $(21,42,66$, and $135 \mathrm{~ms})$, intermediate when neither word was in purple $(33,49,64$, and $101 \mathrm{~ms}$ ), and smallest when the noncolor word was in purple $(12,29,32$, and $55 \mathrm{~ms})$. These distributional differences are consistent with the hypothesis that attention was drawn more often to the word displayed in purple.

$P E$ The main effect of colored word did not reach significance, $F(1,9)<1.0$, but that of congruency did, $F(1,9)=$ $8.31, M S E=8.32, p=.0181$. Responses were more accurate when the color word was congruent with the color of the color bar $(M=0.22 \%)$ than when it was incongruent $(M=$ $2.37 \%$ ), showing a $2.15 \%$ Stroop effect. Unlike with the RT data, the Stroop effect tended to be smaller when no word was presented in purple $(0.67 \%)$ than when either word was (2.73\% for purple color words and 3.04\% for purple neutral words), although this interaction did not approach significance, $F(2,18)=2.03, M S E=4.04, p=.1601$.

\section{Discussion}

In the present experiment, a color bar was always presented at fixation as the color carrier, with a color word and a neutral word as distractors, to increase the discriminability of the target from the distractors. According to Roberts and Besner's (2005) domainspecific limited-capacity account, the Stroop effect should have been of constant size regardless of which word was presented in purple, because the neutral word and colorword distractors were from the same, alphabetic domain and the color carrier was from a different domain. Also, because two stimuli in the same domain were presented as distractors, color-word recognition should have been degraded. However, the Stroop effect was largest when the color word was presented in purple and smallest when the neutral word was presented in purple. When the color word was presented in purple, according to Cho et al.'s (2006) account, the probability of its capturing visual attention increased, resulting in a larger Stroop effect. In contrast, when the neutral word was presented in purple, the probability of the color word capturing visual attention decreased, resulting in a smaller Stroop effect. These results imply that the Stroop effect is modulated by the probability of visual attention being allocated to the color word.

\section{General discussion}

The Stroop dilution effect shows that color-word recognition is degraded when a neutral word is presented as a distractor (e.g., Brown et al., 1995; Kahneman \& Chajczyk,
1983) or as the color carrier (e.g., Cho et al., 2006; Roberts \& Besner, 2005). To explain the Stroop dilution effect, Roberts and Besner suggested that the color word is not processed when a neutral word is presented as the color carrier at fixation, because material-specific attentional resources for processing alphabetic characters are not available for recognition of the color word. That is, the attentional resources are devoted to processing the neutral-word color carrier presented at fixation. However, Cho and colleagues (Cho et al., 2006; Kim, Cho, Yamaguchi, \& Proctor, 2008) suggested that color-word recognition is degraded because the probability of the color word capturing visual attention after processing of the color carrier decreases when a neutral word is presented as a color carrier or distractor.

In the present study, we examined in three experiments whether the allocation of visual attention is responsible for the Stroop dilution effect. When the color of the color word, presented above or below the color carrier as a distractor, varied randomly in Experiment 1, dilution of the Stroop effect was evident if the color word was presented in white, and but not if it was presented in purple. A similar reduction in the Stroop dilution effect for purple color words was evident when the achromatic distractor color was a shade of gray equated for luminance with the purple color. The results of Experiment 1 imply that, even though a neutral word was presented at fixation as the color carrier, the extent to which the color word was recognized increased when it was presented in purple rather than white, lessening dilution of the Stroop effect by the neutral word. Moreover, when the color carrier was a neutral word, a larger Stroop effect tended to be obtained with the color word presented in purple rather than white, indicating that there was competition for attention between the color carrier and the color word presented in purple, which was similar to the possible target colors.

When the color of the color word remained constant throughout Experiment 2, the Stroop dilution effect was evident regardless of the color of the color word. That is, when the color word loses its ability to compete for visual attention because of the invariance of its color (MoreinZamir et al., 2002), the color word appears to be processed after the color carrier is, regardless of the color of the color word. In Experiment 3, when a bar was presented as the color carrier with a color word and a neutral word as distractors, one or none of which was presented in purple, the Stroop effect was modulated by which word was in purple. As Cho et al. (2006) suggested, the largest Stroop effect was obtained when the color word was presented in purple, where the probability of the color word being attended was highest, and the smallest Stroop effect was obtained when the neutral word was presented in purple, where the probability of the color word being attended was lowest. These results imply that because processing of the color word benefits from visual attention, the 
magnitude of the Stroop effect is modulated by the color of a distractor.

\section{Attentional capture account}

Because, in a Stroop task, participants are asked to name (or respond to) the color of the color carrier and to ignore the other stimulus information, they need to pay attention endogenously to the color carrier in order to respond appropriately (see, e.g., Allport, 1989). Even though there is no intention to process the color word, it affects the participant's response to the color (Brown et al., 1995; Neely \& Kahan, 2001; see MacLeod, 1991, for review). As noted, this involuntary aspect of the Stroop effect has been taken to suggest that the color word is automatically recognized in the Stroop task (Brown, Gore, \& Carr, 2002; Moors \& De Houwer, 2006). However, since Kahneman and Chajczyk's (1983) first demonstration that the Stroop effect is reduced when a neutral word is presented as a distractor along with the color word, many researchers have suggested that attention modulates the Stroop effect (e.g., Besner, Stolz, \& Boutilier, 1997; MacLeod \& Bors, 2002; Mitterer, La Heij, \& Van der Heijden, 2003). For example, Kahneman and Chajczyk proposed that Stroop interference is reduced because the color and neutral words compete for attention. According to their account, when the color word captures attention, the color word influences participants' responses to the color. However, when the neutral word captures attention, the color word has no effect on performance.

Cho et al. (2006) claimed that visual attention is most likely oriented initially to the color carrier by the top-down mechanism of control of visual attention because the color carrier is the only task-relevant stimulus, and by the bottomup mechanism because it is the most salient stimulus in the Stroop display. Thus, according to Cho et al., the color word always captures visual attention when it is presented as a color carrier. Consistently, a larger Stroop effect has been obtained when the target color and color word are integrated than when they are separated (MacLeod, 1998). The finding that the size of the Stroop effect is not modulated by the location of the color carrier when the color and color word are presented separately (Cho et al., 2006) indicates that the color carrier has priority for visual attention. Also, when the color word is presented as the color carrier, the presence of a neutral word does not reduce the Stroop effect (Cho et al., 2006; Roberts \& Besner, 2005), because in that case whether a neutral word is present does not change the probability of the color word being attended.

When a color word is presented as a distractor, the probability of the color word capturing visual attention depends on several factors. Cho et al.'s (2006) account assumes that visual attention is oriented to the color carrier because of the outcomes of preattentive processes, especially those that involve the target-defining feature of the color that determines which response to make. In most cases, the color carrier is presented in a target color and the distractors, including a color word, are presented in an achromatic color (white or black). Consequently, the color carrier is most likely to capture visual attention initially. Initial capture by the carrier would also be likely if the set of possible target colors involved different color-feature maps (e.g., Wolfe, 1994) than those activated by the color in which the color word was displayed (e.g., red and blue target set, with the color word presented in yellow). In such cases, the color word could be processed when an attentional shift from the color carrier to the color word occurred after processing of the color carrier. Thus, the probability of the color word receiving visual attention is affected by many factors, including the nature of the color carrier (Cho et al., 2006; Kim et al., 2008; Roberts \& Besner, 2005) and the presence of a neutral word as a distractor (Kahneman \& Chajczyk, 1983). Any factor that affects where attention is directed should influence the Stroop effect.

If the distractors are perceptually similar to the color carrier, attentional competition is expected between them because the distractor is more likely to attract attention. Lamers and Roelofs (2007) showed that the Stroop effect was smaller when the target color was discriminated from the color word by gestalt grouping principles than when it was not. This result indicates that discriminability of the color carrier from the color word can affect initial orienting to the color carrier. In the present experiments, when a distractor was presented in a color (purple) similar to the set of potential target colors, there would be competition for visual attention between the color carrier and the distractor, resulting in an increased probability of the color word capturing visual attention (Exp. 1). It should be noted that attentional competition between the color carrier and a colored distractor occurred only when the color of the distractor varied randomly. This outcome is in agreement with prior findings that a distractor dimension has little or no influence on task performance when that dimension is fixed at a constant value throughout a trial block (e.g., Garner, 1974; Melara \& Mounts, 1993).

\section{Material-specific limited-capacity account}

As mentioned, Roberts and Besner's (2005) materialspecific limited-capacity account differs from Cho et al.'s (2006) account in a couple of ways. First, Roberts and Besner's account assumes that the color carrier presented at fixation has attentional priority. They conducted a series of experiments in which the color carrier was presented at fixation with the color word located either above or below it, to investigate whether the extent to which the color word is recognized is due to the color carrier interfering perceptually with the processing of the color word at an early visual 
processing stage, as suggested by Brown, Roos-Gilbert, and Carr (1995). The Stroop effect was modulated by the stimulus at fixation, indicating that visual attention is required for color-word recognition in the Stroop task. However, because Roberts and Besner's account does not consider the control of visual attention, it has no mechanism to explain why the color carrier at fixation has attentional priority. Thus, its prediction is inconsistent with our findings that the Stroop effect is modulated by the colors of the distractors when the color carrier is constant.

The second difference is that Roberts and Besner's (2005) account assumes that color-word recognition depends on the attentional resources available for processing the word. It is still unclear whether the reduced Stroop effect obtained when the color carrier is a neutral word relative to when it is a bar is due to the limited capacity of a materialspecific attentional resource, as they propose. Even if this is the case, an additional assumption must be made to explain why the Stroop effect is modulated by factors other than the alphabetic/nonalphabetic nature of the color carrier, such as the temporal aspects of the color-word presentation (Cho et al., 2006; Kim et al., 2008).

\section{Conclusion}

When the likelihood of the color word attracting visual attention was manipulated in Experiment 1 by randomly varying the color of a distractor, the Stroop dilution effect was less evident when the color word was presented in purple rather than in white (or, in the follow-up experiment, gray). In Experiment 3, the Stroop effect was larger with a color word presented in purple and a neutral word presented in white than with a color word presented in white and a neutral word presented in purple. These results imply that visual attention is more likely to be oriented to a distractor presented in a color similar to potential target colors than to a distractor presented in white. This finding implies that processing of the color word depends on the likelihood of that word capturing attention, rather than on whether the material of the color carrier at fixation is alphabetic or nonalphabetic. The nature of the color carrier plays a critical role, most likely through decreasing the chance that attention will be directed to the color word as color-carrier processing time increases.

Author Note We thank Ulrich Ansorge and Colin MacLeod for detailed comments on earlier drafts that helped improve the manuscript. This research was supported in part by Korea Research Foundation Grant NRF-2011-327-H00039 and by Korea Science and Engineering Foundation (KOSEF) Grant 20110030099 funded by the Korean government.

\section{References}

Allport, A. (1989). Visual attention. In M. I. Posner (Ed.), Foundations of cognitive science (pp. 631-682). Cambridge: MIT.

Ansorge, U., \& Heumann, M. (2004). Peripheral cuing by abrupt-onset cues: The influence of color in S-R corresponding conditions. Acta Psychologica, 116, 115-143. doi:10.1016/j.actpsy.2004.01.001

Ansorge, U., Horstmann, G., \& Carbone, E. (2005). Top-down contingent capture by color: Evidence from RT distribution analyses in a manual choice reaction task. Acta Psychologica, 120, 243-266. doi:10.1016/j.actpsy.2005.04.004

Becker, S. I., Folk, C. L., \& Remington, R. W. (2010). The role of relational information in contingent capture. Journal of Experimental Psychology: Human Perception and Performance, 36, 1460-1476. doi:10.1037/a0020370

Besner, D., Stolz, J. A., \& Boutilier, C. (1997). The Stroop effect and the myth of automaticity. Psychonomic Bulletin \& Review, 4, 221-225. doi:10.3758/BF03209396

Brown, T. L., Gore, C. L., \& Carr, T. H. (2002). Visual attention and word recognition in Stroop color naming: Is word recognition "automatic"? Journal of Experimental Psychology: General, 131, 220-240. doi:10.1037/0096-3445.131.2.220

Brown, T. L., Roos-Gilbert, L., \& Carr, T. H. (1995). Automaticity and word perception: Evidence from Stroop and Stroop dilution effects. Journal of Experimental Psychology: Learning, Memory, and Cognition, 21, 1395-1411. doi:10.1037/0278-7393.21.6.1395

Cho, Y. S., Lien, M.-C., \& Proctor, R. W. (2006). Stroop dilution depends on the nature of the color carrier but not on its location. Journal of Experimental Psychology: Human Perception and Performance, 32, 826-839. doi:10.1037/0096-1523.32.4.826

Choi, J. M., Cho, Y. S., \& Proctor, R. W. (2009). Impaired color word processing at an unattended location: Evidence from a Stroop task combined with inhibition of return. Memory \& Cognition, 37, 935-944. doi:10.3758/MC.37.6.935

Dalrymple-Alford, E. C., \& Budayr, B. (1966). Examination of some aspects of the Stroop color-word test. Perceptual and Motor Skills, 23, 1211-1214.

Duncan, J., \& Humphreys, G. W. (1989). Visual search and stimulus similarity. Psychological Review, 96, 433-458. doi:10.1037/0033295X.96.3.433

Flowers, J. H., \& Dutch, S. (1976). The use of visual and name codes in scanning and classifying colors. Memory \& Cognition, 4, 384-390.

Folk, C. L., \& Remington, R. (1998). Selectivity in distraction by irrelevant featural singletons: Evidence for two forms of attentional capture. Journal of Experimental Psychology: Human Perception and Performance, 24, 847-858. doi:10.1037/0096-1523.24.3.847

Folk, C. L., Remington, R. W., \& Johnston, J. C. (1992). Involuntary covert orienting is contingent on attentional control settings. Journal of Experimental Psychology: Human Perception and Performance, 18, 1030-1044. doi:10.1037/0096-1523.18.4.1030

Folk, C. L., Remington, R. W., \& Wright, J. H. (1994). The structure of attentional control: Contingent attentional capture by apparent motion, abrupt onset, and color. Journal of Experimental Psychology: Human Perception and Performance, 20, 317-329. doi:10.1037/0096-1523.20.2.317

Francolini, C. M., \& Egeth, H. E. (1979). Perceptual selectivity is task dependent: The pop-out effect. Perception \& Psychophysics, 25, 99-110.

Garner, W. R. (1974). The processing of information and structure. Potomac: Erlbaum.

Glaser, M. O., \& Glaser, W. R. (1982). Time course analysis of the Stroop phenomenon. Journal of Experimental Psychology: Human Perception and Performance, 8, 875-894. doi:10.1037/00961523.8.6.875 
Herd, S. A., Banich, M. T., \& O’Reilly, R. C. (2006). Neural mechanisms of cognitive control: An integrative model of Stroop task performance and fMRI data. Journal of Cognitive Neuroscience, 18, 22-32. doi:10.1162/089892906775250012

Johnson, A., \& Proctor, R. W. (2004). Attention: Theory and practice. Thousand Oaks: Sage.

Kahneman, D., \& Chajczyk, D. (1983). Tests of the automaticity of reading: Dilution of Stroop effects by color-irrelevant stimuli. Journal of Experimental Psychology: Human Perception and Performance, 9, 497-509. doi:10.1037/0096-1523.9.4.497

Kiesel, A., Steinhauser, M., Wendt, M., Falkenstein, M., Jost, K., Philipp, A. M., \& Koch, I. (2010). Control and interference in task switching-a review. Psychological Bulletin, 136, 849-874. doi:10.1037/a0019842

Kim, H., Cho, Y. S., Yamaguchi, M., \& Proctor, R. W. (2008). Influence of color word availability on the Stroop color-naming effect. Perception \& Psychophysics, 70, 1540-1551. doi:10.3758/PP.70.8.1540

Lachter, J., Forster, K. I., \& Ruthruff, E. (2004). Forty-five years after Broadbent (1958): Still no identification without attention. Psychological Review, 111, 880-913. doi:10.1037/0033-295X.111.4.880

La Heij, W., Helaha, D., \& Van den Hof, E. (1993). Why does blue hamper the naming of red? Color-color interference and the role of locational (un)certainty. Acta Psychologica, 83, 159-177. doi:10.1016/0001-6918(93)90052-S

Lamers, M. J. M., \& Roelofs, A. (2007). Role of Gestalt grouping in selective attention: Evidence from the Stroop task. Perception \& Psychophysics, 69, 1305-1314. doi:10.3758/BF03192947

Lavie, N. (2005). Distracted and confused? Selective attention under load. Trends in Cognitive Sciences, 9, 75-82. doi:10.1016/j. tics.2004.12.004

Lavie, N., \& Fox, E. (2000). The role of perceptual load in negative priming. Journal of Experimental Psychology: Human Perception and Performance, 26, 1038-1052. doi:10.1037/00961523.26.3.1038

MacLeod, C. M. (1991). Half a century of research on the Stroop effect: An integrative review. Psychological Bulletin, 109, 163203. doi:10.1037/0033-2909.109.2.163

MacLeod, C. M. (1998). Training on integrated versus separated Stroop tasks: The progression of interference and facilitation. Memory \& Cognition, 26, 201-211.

MacLeod, C. M., \& Bors, D. A. (2002). Presenting two color words on a single Stroop trial: Evidence for joint influence, not capture. Memory \& Cognition, 30, 789-797. doi:10.3758/ BF03196434

MacLeod, C. M., \& Dunbar, K. (1988). Training and Stroop-like interference: Evidence for a continuum of automaticity. Journal of Experimental Psychology: Learning, Memory, and Cognition, 14, 126-135. doi:10.1037/0278-7393.14.1.126

Melara, R. D., \& Mounts, J. R. W. (1993). Selective attention to Stroop dimensions: Effects of baseline discriminability, response mode, and practice. Memory \& Cognition, 21, 627-645. doi:10.3758/ BF03197195

Mitterer, H., La Heij, W., \& Van der Heijden, A. H. C. (2003). Stroop dilution but not word-processing dilution: Evidence for attention capture. Psychological Research, 67, 30-42.

Moors, A., \& De Houwer, J. (2006). Automaticity: A theoretical and conceptual analysis. Psychological Bulletin, 132, 297-326. doi:10.1037/0033-2909.132.2.297

Morein-Zamir, S., Henik, A., \& Spitzer-Davidson, I. (2002). The importance of irrelevant-dimension variability in the Stroop flanker task. Psychonomic Bulletin \& Review, 9, 119-125.

Neely, J. H., \& Kahan, T. A. (2001). Is semantic activation automatic? A critical re-evaluation. In H. L. Roediger III, J. S. Nairne, I. Neath, \& A. M. Surprenant (Eds.), The nature of remembering: Essays in honor of Robert G. Crowder (pp. 69-93). Washington: American Psychological Association. doi:10.1037/10394-005

Proctor, R. W., Miles, J. D., \& Baroni, G. (2011). Reaction time distribution analysis of spatial correspondence effects. Psychonomic Bulletin \& Review, 18, 242-266. doi:10.3758/s13423011-0053-5

Roberts, M. A., \& Besner, D. (2005). Stroop dilution revisited: Evidence for domain-specific, limited-capacity processing. Journal of Experimental Psychology: Human Perception and Performance, 31, 3-13. doi:10.1037/0096-1523.31.1.3

Stroop, J. R. (1992). Studies of interference in serial verbal reactions. Journal of Experimental Psychology: General, 121, 15-23. doi:10.1037/0096-3445.121.1.15. Original work published 1935 .

Theeuwes, J. (1992). Perceptual selectivity for color and form. Perception \& Psychophysics, 51, 599-606. doi:10.3758/BF03211656

Wolfe, J. M. (1994). Guided Search 2.0: A revised model of visual search. Psychonomic Bulletin \& Review, 1, 202-238. doi:10.3758/ BF03200774 